\title{
An 83-year-old-male with bronchopleural fistula and empyema successfully treated with multidisciplinary management of thoracostomy, endoscopic, and surgical treatment: a case report
}

\author{
Zhongliang $\mathrm{He}^{1}$, Lifeng Shen ${ }^{2}$, Weihua $\mathrm{Xu}^{3}$, Xiaowen $\mathrm{He}^{4}$ \\ ${ }^{1}$ Department of Cardiothoracic Surgery, Tongde Hospital of Zhejiang Province, Hangzhou, China; ${ }^{2}$ Division of Repairing and Reconstructive \\ Centre, Department of Orthopedics, Tongde Hospital of Zhejiang Province, Hangzhou, China; ${ }^{3}$ Department of Interventional Pulmonology, \\ Tongde Hospital of Zhejiang Province, Hangzhou, China; ${ }^{4}$ Division of Endocrinology and Metabolism, Department of Medicine, Zhejiang \\ University Medical School Second Affiliated Hospital, Hangzhou, China \\ Correspondence to: Xiaowen He. Division of Endocrinology and Metabolism, Department of Medicine, Zhejiang University Medical School Second \\ Affiliated Hospital, 88 Jiefang Rd, Hangzhou 310020, China. Email: $2185008 @$ zju.edu.cn.
}

\begin{abstract}
Bronchopleural fistula (BPF) with empyema is a severe complication in patients undergoing lobectomy or pneumonectomy and is associated with high morbidity and mortality rates. Although a wide variety of treatment options exist, refractory cases with larger fistulas are still difficult to cure, especially in elderly patients. Here, we report a case of an 83-year-old man with stage I squamous cell lung carcinoma who underwent minimally invasive right lower lobectomy. After an initially uneventful postoperative course, he was readmitted to our hospital due to the progression of severe cough with fever after lung resection. Chest computed tomography (CT) showed an empyema cavity containing pleural effusion and a drainage tube in the right lower thorax. Bronchoscopy confirmed the presence of a fistula between the right lower bronchial stump and the pleural cavity. On the basis of his clinical symptoms and these imaging findings, the patient was diagnosed with BPF with empyema after lobectomy. He was successfully treated with multidisciplinary management including adequate pleural drainage by open-window thoracostomy, closure of the BPF by endoscopic therapy using an Amplatzer device, and complete obliteration of the empyema cavity with pedicled muscle flap. Multidisciplinary management combining thoracostomy, endoscopic therapy, and pedicled muscle flap transfer is a safe and effective treatment for elderly patients with larger fistulas and empyema.
\end{abstract}

Keywords: Bronchopleural fistula (BPF); empyema; endoscopy; muscle flap; case report

Submitted Apr 01, 2020. Accepted for publication Dec 06, 2020.

doi: $10.21037 /$ atm-20-3053

View this article at: http://dx.doi.org/10.21037/atm-20-3053

\section{Introduction}

Bronchopleural fistula (BPF) with empyema is an uncommon but severe complication in patients undergoing lobectomy or pneumonectomy, and has rates of high morbidity and mortality $(1,2)$. Successful management of BPF remains challenging due to difficulties relating to infection control, and frequent, easy redevelopment of residual space and fistula. Despite the wide variety of treatment options for BPF, curing refractory cases of larger fistulas is still difficult, especially in elderly patients $(3,4)$. In this report, we describe the case of an 83-year-old male with BPF and empyema who was successfully treated with multidisciplinary management including open-window thoracostomy, endoscopic Amplatzer device placement, and pedicled muscle flap transfer. We present the following case in accordance with the CARE reporting checklist (available at http://dx.doi.org/10.21037/atm-20-3053).

\section{Case presentation}

All procedures performed in studies involving human participants were in accordance with the ethical standards 


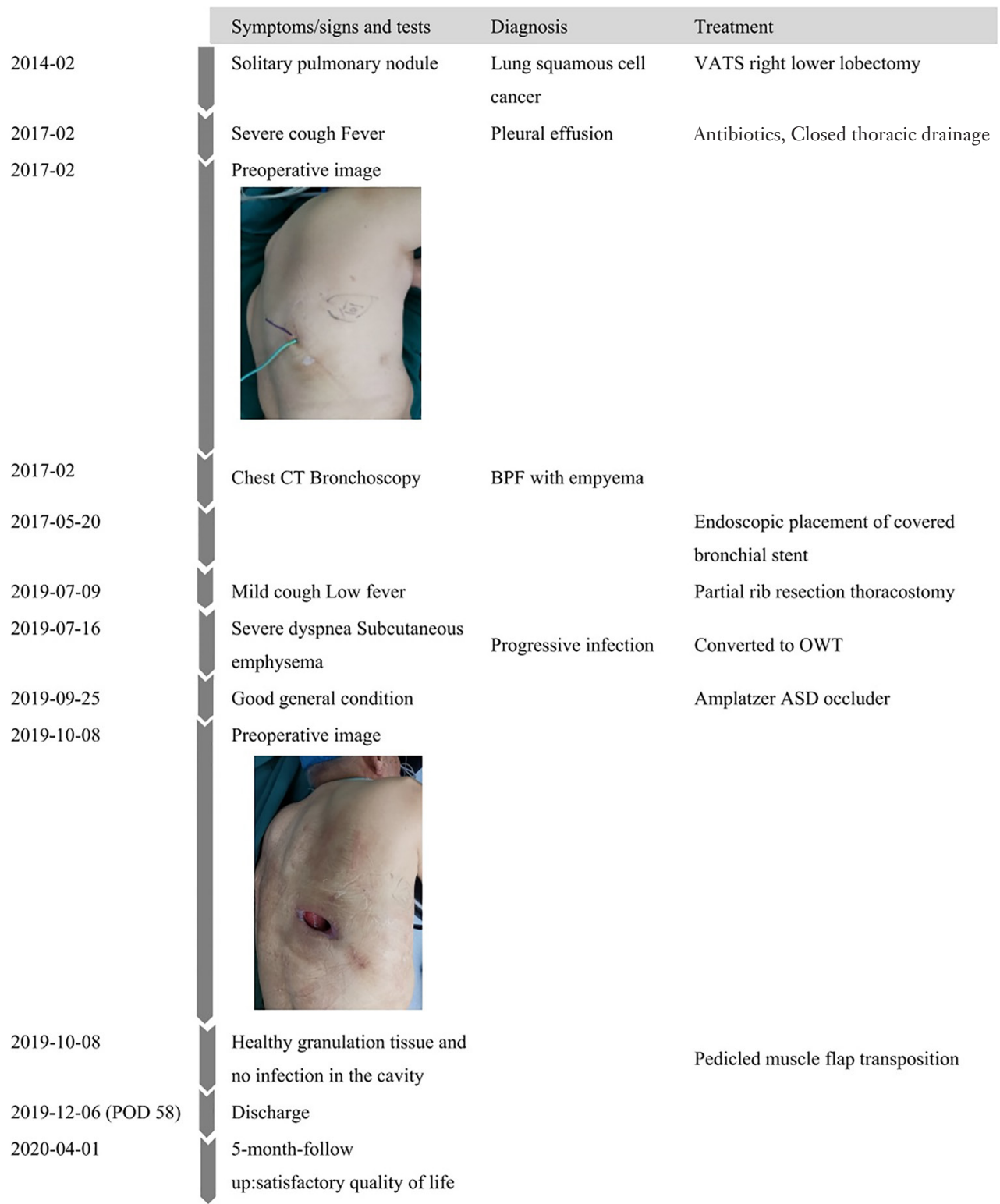

Figure 1 Timeline of the patient's main symptoms, diagnoses, and treatments. VATS, video-assisted thoracoscopic surgery; CT, computed tomography; BPF, bronchopleural fistula; OWT, open window thoracostomy; ASD, atrial septal defect; POD, postoperative day.

of the institutional and national research committees, and with the Declaration of Helsinki (as revised in 2013). Written informed consent was obtained from the patient for publication of this manuscript and any accompanying images.

A healthy 83 -year-old man with a solitary pulmonary nodule, later identified as pT1bN0M0 lung squamous cell carcinoma, underwent minimally invasive right lower lobectomy in February 2014 (Figure 1). Three years after undergoing lung resection, the patient was admitted to our hospital due to the progression of severe cough with fever. Although the patient's fever was relieved by the administration of antibiotics and insertion of closed thoracic drainage, his cough continued, and a large air leak from the tube was observed (Figure 1). Chest computed tomography (CT) showed an empyema cavity measuring $9 \mathrm{~cm} \times 7 \mathrm{~cm} \times$ $6 \mathrm{~cm}$ containing pleural effusion as well as a drainage tube in the right lower thorax. Bronchoscopy confirmed the 

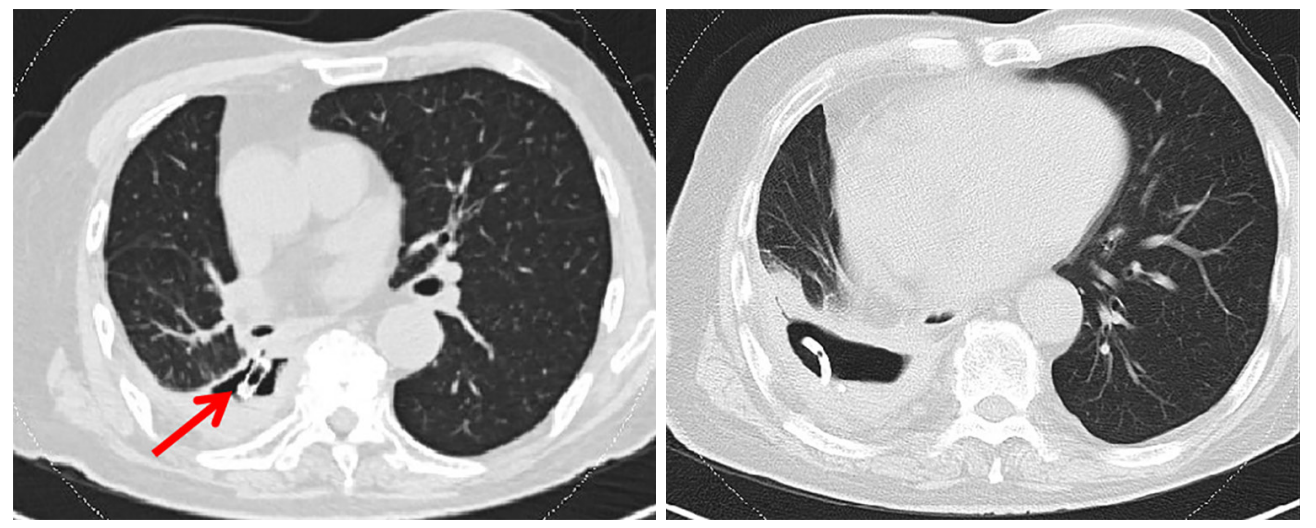

Figure 2 Preoperative chest CT scan showing an empyema cavity measuring $9 \mathrm{~cm} \times 7 \mathrm{~cm} \times 6 \mathrm{~cm}$ containing pleural effusion as well as the tube and the covered bronchial stent (arrow) in the right lower thorax. CT, computed tomography.

presence of a fistula of approximately $7 \mathrm{~mm}$ in diameter between the right lower bronchial stump and the pleural cavity.

On the basis of his clinical symptoms and imaging findings, the patient was diagnosed with right lower BPF with empyema after lobectomy, and he subsequently underwent endoscopic placement of a covered bronchial stent (Boston Scientific Corporation, Natick, MA, USA) in May 2017. Thereafter, the cough decreased, and the air leak from the tube was reduced. However, 1 week later, air leakage and purulent fluid discharge from the tube increased again, and further investigation with chest CT revealed that the stent had migrated into the empyema cavity (Figure 2). On April 7, 2020, due to the inadequate results, the patient underwent rib resection thoracostomy; the posterior aspects of the $7^{\text {th }}$ and $8^{\text {th }}$ ribs were resected. The stent and purulent pleural effusion in the thoracic cavity were removed by evacuation and debridement, and positive-pressure ventilation revealed a large air leakage. Then, 2 drainage tubes were inserted into the cavity, the wound was closed, and the cavity was irrigated with an antibiotic solution through the tubes. Culturing of the drained pus identified the pathogens Klebsiella pneumoniae and Pseudomonas aeruginosa. Seven days later, he began to experience marked dyspnea, and subcutaneous emphysema developed; his closed drainage was subsequently converted to open-window thoracostomy (Figure 1), with regular dressing changes and application of a compressive bandage during the following months. After his general condition had improved, the patient underwent re-endoscopic therapy, in which an Amplatzer atrial septal defect occluder (Shandong Visee Medical Devices Co., Ltd., Shandong,
China) was placed between the proximal and distal ends of the stump to almost completely close the fistula from both sides of the defect with its central waist and 2 discs of both ends. The large air leakage was markedly reduced with drainage, and the patient's condition gradually stabilized.

When healthy granulation tissue appeared and culture detected no infection in the thoracic cavity, muscle flap transposition was performed. During the operation, a $20 \mathrm{~cm} \times 15 \mathrm{~cm}$ combined pedicled muscle flap of the latissimus dorsi and serratus anterior muscles was harvested. The tip of the combined flap was sutured and fixed to the mid-lower mediastinum, and the remainder was transposed to almost completely obliterate the empyema cavity, while part of the sacrospinalis muscle was transferred to fill the posterior residual space (Figure $3 A$ ). The patient was discharged on postoperative day 58 (Figure 3B). Chest CT scan after surgery revealed that the BPF and empyema cavity had been successfully obliterated (Figure 4). The patient has remained healthy, and no recurrence of fistula with empyema was evident at the 5-month follow-up.

\section{Discussion}

The cause of $\mathrm{BPF}$ is abnormal communication between the pleural space and the bronchial tree. BPF is a potentially fatal postoperative complication of pulmonary resection and a complex challenge for thoracic surgeons, as many patients with fistula ultimately have refractory empyema. Managing refractory empyema is difficult, especially in elderly individuals, and the quality of life and survival of patients are severely impacted $(1,2)$. BPF has a prevalence of $1.5-28 \%$ after pulmonary resection, and this variability 

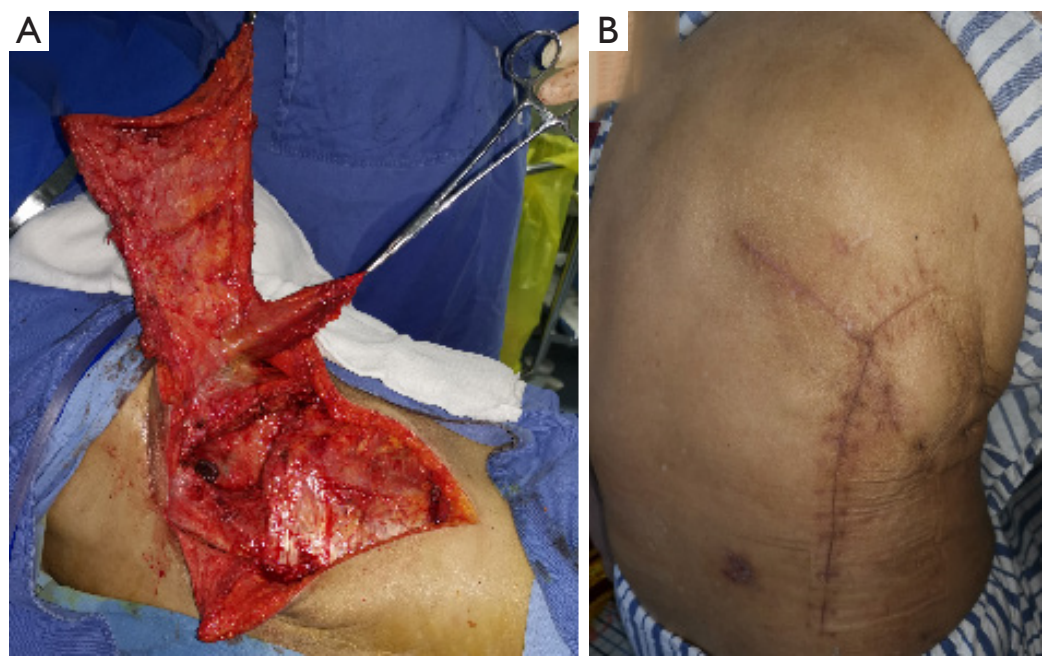

Figure 3 Operative and postoperative images. (A) Pedicled latissimus dorsi and serratus anterior muscle flaps. (B) The thoracic incision healed extremely well.
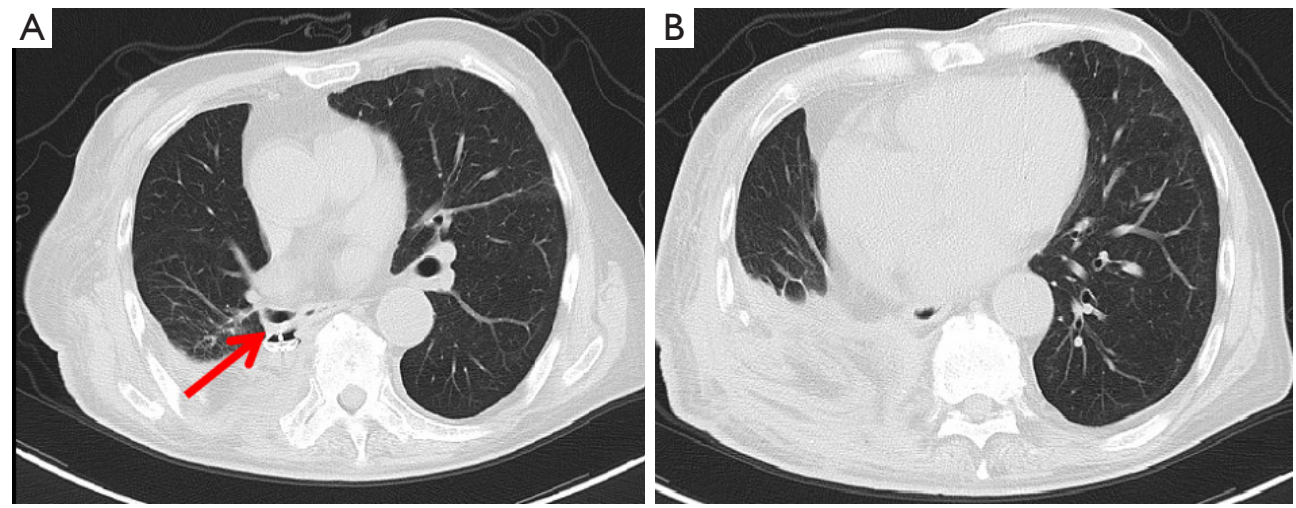

Figure 4 Postoperative chest CT scan at the 5-month follow-up revealing successful obliteration of the BPF and empyema cavity with an Amplatzer device (arrow) and combined pedicled muscle flap. CT, computed tomography; BPF, bronchopleural fistula.

seemingly depends on the etiology, surgical technique, and experience of the surgeon $(3,4)$.

The etiology of BPF with empyema is still not fully understood. Local risk factors include stump closure technique, a long bronchial stump, residual carcinoma at the bronchial margin, disruption of the bronchial blood supply, extended resection, right-side resection, pneumonectomy, the presence of empyema, and high-dose preoperative radiation therapy, although no single factor has been definitively identified. Systemic factors include the patient's nutritional status, diabetes mellitus, steroid use, the presence of sepsis, and preoperative chemotherapy $(5,6)$. BPF typically presents 1 to 2 weeks after lung resection, manifesting as fever, productive cough, purulent or hemorrhagic sputum, respiratory distress, and occasionally as sepsis and acute respiratory failure. Patients typically subacutely develop malaise, flu-like symptoms, lowgrade fever, and weight loss, which can lead to persistent contamination and infection of the pleural space, trapped lung, aspiration in the unaffected lung, and in severe cases, death. The mortality rate associated with BPF after pneumonectomy has been reported to be $20-70 \%(5,6)$. Diagnosis of BPF is usually confirmed by bacteriological study, chest CT scan, or bronchoscopy.

Following the development of BPF with empyema, proper and prompt management is essential to reduce 
the risk of associated mortality. Curing larger BPFs in elderly patients is still challenging, despite the wide variety of treatment options. Management options include thoracostomy, endoscopy, surgery, or a combination of methods, and the selection of a particular therapy is based on clinical status, the size of the BPF, and acuity of the empyema (2-6). Adequate pleural drainage remains the cornerstone of empyema treatment. If BPF with empyema is diagnosed, it should be drained promptly to prevent an aspiration pneumonia, life-threatening sequelae such as tension pneumothorax, and aspiration and respiratory failure. Closed chest tube drainage has been advocated as the first step in the treatment of BPF with empyema; however, with a high failure rate, chest tube drainage cannot control BPF with empyema effectively and increases the risk of contralateral lung inhalation and death. Openwindow thoracostomy has proven very useful in the treatment of $\mathrm{BPF}$ and is credited with saving many lives. It is a simple technique that may be performed even on extremely unstable patients, like the patient in this case study $(4,5)$. Endoscopic therapy should be performed to promote fistula closure after thoracic drainage, as it is simple, safe, and less invasive compared to surgical treatment. Larger BPFs cannot be treated using endoscopic techniques, since neither coils nor fibrin glue is suitable for large bronchial stump fistulas due to insufficient stability in the lesion. Despite this, many researchers have shown that Amplatzer devices are suitable for the closure of fistulas of different sizes that cannot be treated successfully with other treatment modalities, especially in high-risk patients with larger fistulas (6-8). However, failure of endoscopic treatment does not preclude subsequent successful surgical management, as it may be used as a bridge to surgical treatment, as was the case in our patient (6).

Muscle flap transfer may be used to obliterate BPF with empyema and may be applied using pedicled, free, and combination methods. Pedicled muscle flaps are ideal for filling a contaminated space due to their good blood supply and ability to reach almost any location in the pleural space. The most common muscles used are the latissimus dorsi, serratus anterior, pectoralis major, pectoralis minor, and intercostals. Among these, the latissimus dorsi muscle flap is the largest and most reliable (9). The proximal part of the muscle can be pedicled on the thoracodorsal vessels or the serratus branch, so it can be elevated at full length and has bulky tissue that provides reliable closure of the BPF with empyema. However, elderly patients with a large pleural cavity or those who are unsuitable for receiving a free muscle flap could have 2 or more combined pedicled muscle flaps transferred simultaneously to obliterate the pleural cavity, like in the case of our patient (9). When a pedicled muscle flap cannot be used, a free musculocutaneous flap harvested from a vastus lateralis or rectus abdominis can be transposed to completely obliterate the cavity (10).

A limitation of this study is that it is a single case report, and the other inherent limitation is its retrospective nature. As a result of these limitations, it may be difficult to glean a firm conclusion from this case. Our patient remained healthy and experienced no recurrence of fistula with empyema for over 5 months following multidisciplinary management.

In conclusion, in patients with $\mathrm{BPF}$, it is crucial to not only completely control infection and occlude the fistula, but also to obliterate the empyema cavity. Therefore, multidisciplinary management combining open-window thoracostomy, endoscopic Amplatzer device placement, and pedicled muscle flap transfer is a useful option for treating older patients with larger fistulas and empyema. Our report indicates that this is a feasible and efficient method of management that can achieve promising results.

\section{Acknowledgments}

Funding: None.

\section{Footnote}

Reporting Checklist: The authors have completed the CARE reporting checklist. Available at http://dx.doi.org/10.21037/ atm-20-3053

Conflicts of Interest: All authors have completed the ICMJE uniform disclosure form (available at http://dx.doi. org/10.21037/atm-20-3053). The authors have no conflicts of interest to declare.

Ethical Statement: The authors are accountable for all aspects of the work in ensuring that questions related to the accuracy or integrity of any part of the work are appropriately investigated and resolved. All procedures performed in studies involving human participants were in accordance with the ethical standards of the institutional and national research committees, and with the Declaration of Helsinki (as revised in 2013). Written informed consent was obtained from the patient for publication of this 
manuscript and any accompanying images.

Open Access Statement: This is an Open Access article distributed in accordance with the Creative Commons Attribution-NonCommercial-NoDerivs 4.0 International License (CC BY-NC-ND 4.0), which permits the noncommercial replication and distribution of the article with the strict proviso that no changes or edits are made and the original work is properly cited (including links to both the formal publication through the relevant DOI and the license). See: https://creativecommons.org/licenses/by-nc-nd/4.0/.

\section{References}

1. Zanotti G, Mitchell JD. Bronchopleural fistula and empyema after anatomic lung resection. Thorac Surg Clin 2015;25:421-7.

2. Hysi I, Rousse N, Claret A, et al. Open window thoracostomy and thoracoplasty to manage 90 postpneumonectomy empyemas. Ann Thorac Surg 2011;92:1833-9.

3. Boudaya MS, Smadhi H, Zribi H, et al. Conservative management of postoperative bronchopleural fistulas. J Thorac Cardiovasc Surg 2013;146:575-9.

4. Zaheer S, Allen MS, Cassivi SD, et al. Postpneumonectomy

Cite this article as: He Z, Shen L, Xu W, He X. An 83-yearold-male with bronchopleural fistula and empyema successfully treated with multidisciplinary management of thoracostomy, endoscopic, and surgical treatment: a case report. Ann Transl Med 2021;9(5):427. doi: 10.21037/atm-20-3053 empyema: results after the Clagett procedure. Ann Thorac Surg 2006;82:279-86.

5. Laperuta P, Napolitano F, Vatrella A, et al. Postpneumonectomy broncho-pleural fistula successfully closed by open-window thoracostomy associated with V.A.C. therapy. Int J Surg 2014;12:S17-9.

6. Cardillo G, Carbone L, Carleo F, et al. The rationale for treatment of postresectional bronchopleural fistula: analysis of 52 patients. Ann Thorac Surg 2015;100:251-7.

7. Motus IY, Bazhenov AV, Basyrov RT, et al. Endoscopic closure of a bronchopleural fistula after pneumonectomy with the Amplatzer occluder: a step forward? Interact Cardiovasc Thorac Surg 2020;30:249-54.

8. Fruchter O, El Raouf BA, Abdel-Rahman N, et al. Efficacy of bronchoscopic closure of a bronchopleural fistula with amplatzer devices: long-term follow-up. Respiration 2014;87:227-33.

9. Lu C, Feng Z, Ge D, et al. Pedicle muscle flap transposition for chronic empyema with persistent bronchopleural fistula: experience of a single clinical center in China. Surg Today 2016;46:1132-7.

10. He X, He Z, Shen L, et al. Free musculocutaneous flap transfer for refractory chronic empyema with chest wall sinus in a 43-year-old male with hemophilia A. J Thorac Dis 2018;10:E416-9. 